\title{
Conflict Mechanism of Humanoid Robots Driven by Emotion
}

\author{
Guofeng Zhang ${ }^{1, a^{*}}$, Beixing $\mathrm{Mao}^{2, \mathrm{~b}}$ and Hubing $\mathrm{Du}^{1, \mathrm{c}}$ \\ ${ }^{1}$ Institute of Behavior Computing, Xi'an Technological University, 710032, Xi'an, China \\ ${ }^{2}$ Department of Mathematics and Physics, Zhengzhou Institute of Aeronautical Industry \\ Management, 450015, Zhengzhou, China \\ azgfwlz@sina.com, bbxmao329@163.com, xhdhub@126.com
}

*The corresponding author

Keywords: Behavior game; Emotion; Coercive bargain; Humanoid robots

\begin{abstract}
Game equilibrium based on mathematical logic can not predict the real game outcome. In order to solve the problem, a fresh game theory based on emotion is suggested by adding emotion mechanism into mathematical logic. The principle is applied to the game analysis of Coercive bargain between Two Humanoid Robots. The obtained emotion equilibriums imply the reasonable explanations for the common rules of behaviors, such as "Leave Adequate Leeway", "Change from the Defensive to the Offensive" and "Seesaw Battle". Therefore, the rationality and validity of the emotional game-playing thought have been verified elementarily.
\end{abstract}

\section{Introduction}

Behavior selection mechanism of humanoid robot includes decision-making and game -playing as of the real human. Adding emotions into the mechanism is a hot topic in recent years [1]. Among these researches, a fresh emotion-driven decision-making mechanism is suggested [2]. The application of this mechanism not only solves the Allais paradox, Two Envelope paradox and St. Petersburg paradox in a new emotional way [3], but also establishes a competitive (single objective decision-making) and a cooperative (multi-objective decision-making) behavior selection mechanism respectively [4,5]. Recently, the principle of fighting behavior is revealed in animals [6]. Decision-making is simpler, compared with the game-playing. Therefore, the literature is more common on its application to decision-making [7]. For this reason, emotions are applied to game-playing; emotion-driven game theory has been suggested [8]. The principle of conflict burst will be explored by the fresh game-playing principle in this paper.

\section{Classic Conflict between Two Humanoid Robots}

Coercive bargaining is a modified ultimatum game, which was used to analyze the conflict behavior [9]. In this paper, an emotional game-playing on it will be analyzed. The game-playing process is as follows (see Fig. 1).



Figure 1. The coercive bargaining game 
The game begins with a decision by Robot $\mathrm{A}$ to make a demand (Demand), or make no demand ( Demand), thereby maintaining the status quo $\left(q_{0}, 1-q_{0}\right)$. If $\mathrm{A}$ makes a demand, Robot B can either accept the demand (Accept) or reject it (Reject). Accepting the demand implies a settlement outcome ( $q$, 1-q), in which A gets what it demanded and B gets the remainder of the pie. If Robot B rejects A's demand, A can either back down ( ${ }^{2}$ Fight) - allowing the status quo to be maintained nevertheless $\left(q_{0}\right.$, $\left.1-q_{0}\right)$ - or force a winner-take-all contest by fighting (Fight).

A considers its probability of winning the contest is $p_{A}$, the fighting costs $c_{A}$; And $\mathrm{B}$ assumes its probability of winning the contest is $p_{B}$, the fighting cost $c_{B} ; e_{c A}, e_{c B}$ is the negative emotion intensity brought about respectively by $c_{A}, c_{B} ; e_{r A}, e_{r B}$, emotion resolutions; $\lambda_{A}, \lambda_{B}$, the sensitive coefficients of negative emotion.

\section{Emotional Game-playing Principle of Coercive Bargaining}

According to literature [8], the basic analysis results are as follows. If the A-side combat threat is credible, its own current share $q_{0}$ should satisfy Eq. 1,

$$
q_{0} \leq q_{0 \max }= \begin{cases}-\frac{\left\{\lambda_{A} w\left(1-p_{A}\right) w\left(p_{A}\right)+\left[w\left(p_{A}\right)-\lambda_{A} w\left(1-p_{A}\right)\right] e_{A}\right\}^{1 / 2}+w\left(p_{A}\right)}{w\left(p_{A}\right)-\lambda_{A} w\left(1-p_{A}\right)} & \delta\left(p_{A}\right) \geq \lambda_{A} \\ \frac{\left\{\lambda_{A} w\left(1-p_{A}\right) w\left(p_{A}\right)+\left[w\left(p_{A}\right)-\lambda_{A} w\left(1-p_{A}\right)\right] e_{A}\right\}^{1 / 2}-w\left(p_{A}\right)}{\lambda_{A} w\left(1-p_{A}\right)-w\left(p_{A}\right)} & \delta\left(p_{A}\right)<\lambda_{A}\end{cases}
$$

where ,

$$
\begin{aligned}
& e_{r A}+e_{c A}=k_{A} e_{A}>0 \\
& \delta\left(p_{A}\right)=\frac{w\left(p_{A}\right)}{w\left(1-p_{A}\right)} .
\end{aligned}
$$

Thus, once Robot A's holding share reaches, even exceeds the value calculated from Eq. 1, its challenge will lose the threat to Robot B.

Robot A's demanding emotion is greater than its fighting emotion, if $q_{d}$, the share held by the claim, meets Eq. 4,

$$
q_{d}>q_{d \min }=1-\left\{\left(1-q_{0}\right)^{2}\left(1-w\left(p_{A}\right)\right)+\lambda_{A} w\left(1-p_{A}\right) q_{0}^{2}+e_{A}^{\prime}\right\}^{1 / 2} .
$$

where ,

$$
e_{r A}-e_{c A}=k_{A} e_{A}^{\prime}>0
$$

Conversely, $q_{d}$ satisfies Eq. 6,

$$
q_{d}<q_{d \max }=1-\left\{\left(1-q_{0}\right)^{2}\left(1-w\left(p_{A}\right)\right)+\lambda_{A} w\left(1-p_{A}\right) q_{0}^{2}+e_{A}\right\}^{1 / 2} .
$$

If Robot A's held share $x_{d}$ will meet the relationship Eq. 7, its request can be accepted by Party B:

$$
x_{d} \leq x_{d \max }=1-\left\{\left(1-w\left(1-p_{B}\right)\right)\left(1-q_{0}\right)^{2}+w\left(p_{B}\right) q_{0}^{2} / \lambda_{B}-e_{B}^{\prime} / \lambda_{B}\right\}^{1 / 2}
$$

In this situation, $\mathrm{B}$ has no choice but to compromise. So, if there exists an equilibrium, i.e., both sides do not conflict with each other, $q_{d \min }<x_{d \max }$ must be set up.

B side must meet the challenge, if A's share $x_{f b}$ meets the relationship Eq. 8 after the request, 


$$
x_{f b}>x_{f b \min }= \begin{cases}\frac{\left[\lambda_{B} w\left(p_{B}\right) w\left(1-p_{B}\right)-\lambda_{B} w\left(1-p_{B}\right) e_{B}+w\left(p_{B}\right) e_{B}\right]^{1 / 2}-\lambda_{B} w\left(1-p_{B}\right)}{w\left(p_{B}\right)-\lambda_{B} w\left(1-p_{B}\right)} & \delta\left(p_{B}\right)>\lambda_{B} \\ \frac{\left\{\lambda_{A} w\left(1-p_{A}\right) w\left(p_{A}\right)+\left[w\left(p_{A}\right)-\lambda_{A} w\left(1-p_{A}\right)\right] e_{A}\right\}^{1 / 2}-w\left(p_{A}\right)}{\lambda_{A} w\left(1-p_{A}\right)-w\left(p_{A}\right)} & \delta\left(p_{B}\right)<\lambda_{B}\end{cases}
$$

where,

$$
\delta\left(p_{B}\right)=\frac{w\left(p_{B}\right)}{w\left(1-p_{B}\right)} .
$$

Robot $\mathrm{B}$ has to select giving up, if the held share by Robot A after his demanding is less than $x_{f b m i n}$, because the emotion of its fighting back is negative. Therefore, on the condition that Eq. 5 holds, Robot A's demanding does not incur B's fighting behavior, as long as $q_{d}<x_{f b m i n}$. Therefore, the game equilibrium $\left(x_{f b m i n}, 1-x_{f b m i n}\right)$ is obtained.

\section{Concrete Results of Emotional Game-playing Equilibrium}

Set the Simulation Parameters. In order to obtain more clearly the concrete results of above analysis, a Matlab simulation have been programmed. The relevant parameters are set as follows.

The game begins, A side is set to hold initial share $q_{0}=0: 0 ; p_{A} \in[0: 1 ; 0: 9]$, is the ability of A's wining the battle, $p_{B}=1-p_{A}$. Their emotion resolution is set respectively: $e_{r A}=e_{r B}=0.01$. And, $\lambda_{A}, \lambda_{B} \in$ $[1: 3 ; 1: 9]$ is set, according to the negative emotion sensitive coefficient $1.0<\lambda<2.0$. The involved $w(p)$ is assumed as Eq. 10 [10], where there are $a=0.4, b=0.5$.

$$
w(p)=\frac{3(1-b)}{a^{2}-a+1}\left(x^{3}-(a+1) p^{2}+a x\right)+p
$$

There are $e_{A}<0.15$ and $e_{B}<0.15$. Therefore, $e_{A}, e_{B}=[0.05,0.10,0.15]$ is taken, respectively, as the low, medium and high conflict cost. Also, there is $k_{A}=k_{B}=1.0$. Based on these parameters, the game simulation has been completed. And the typical results obtained are shown in Fig. 2-Fig. 3.

"Leave Adequate Leeway". It can be found the demanding process is carried out only once even though A's combat capability is high. This is not acceptable for the wiser if the obtained share after one demanding is less than the obtained share during his two times of demanding. To avoid the outcome, A can demand $q_{d}$, which is smaller than $q_{0 \max }$, on the premise that $q_{d}$ is bigger than $q_{d \max }$. This is namely "leave adequate leeway", one Chinese ancient proverb on behavior strategy. This objective can be achieved when an emotional resolution is added in the fighting emotion. In this way, Eq.5 is changed into Eq.11.

$$
2 e_{r A}+e_{c A}=k_{A} e_{A}{ }^{\prime \prime}>0
$$

To distinguish from the parameters of pervious formulae, $q_{d l}$ is used rather than $q_{d}$, where $e_{A}^{\prime}$ is replaced by $e_{A}$ ".

After two demanding, if the share held by Party A would be greater than or equal to the one accepted by Part B's fighting back behavior, Party A should decrease his demanding quantity in order to avoid the coming conflict. At this point, A can forecasts his owned share after his second demanding. This is to say, B's fighting emotion must be less than his emotion resolution. Thus, $q_{d e}$ is obtained, where the $e_{B}$ in Eq.8 is substituted for $e_{B}{ }^{\prime}$.

One more simulation was completed, $q_{d}, q_{d e}$ were taken into consideration. The simulation results are shown in Fig. 2.

At the first demand, it is also found that Robot A must "leave adequate leeway" in order to get a second chance to demand. The first demand is shown as '*' line in Fig. 2. On the second demand, when A's fighting ability $p$ is large, the amount of his request would exceed the B's fighting back line. Robot A would be bound to encounter in B's counterattack. To avoid conflict, the obtained value can only be 
as $q_{d e}$. Here, it can be seen that the fighting back behavior is taken by the weaker at his small held amount, which is the "Anti-Defensive to the Offensive".

"Seesaw Battle". For $p_{A} \neq 1-p_{B}$, on the condition that $\mathrm{e}_{A}=0.05, e_{B}=0.10, \lambda_{A}=1.9, \lambda_{B}=1.3$, the simulation results are shown in Fig.3. Where $p_{A}>0.6, p_{B}>0.6$, there is $x_{d \max }<q_{d \max }$. In such a situation, A's fighting emotion is greater than his demanding emotion, and B's fighting emotion is also greater than his compromising emotion. Therefore, the conflict is inevitable. After the conflict, the loser will

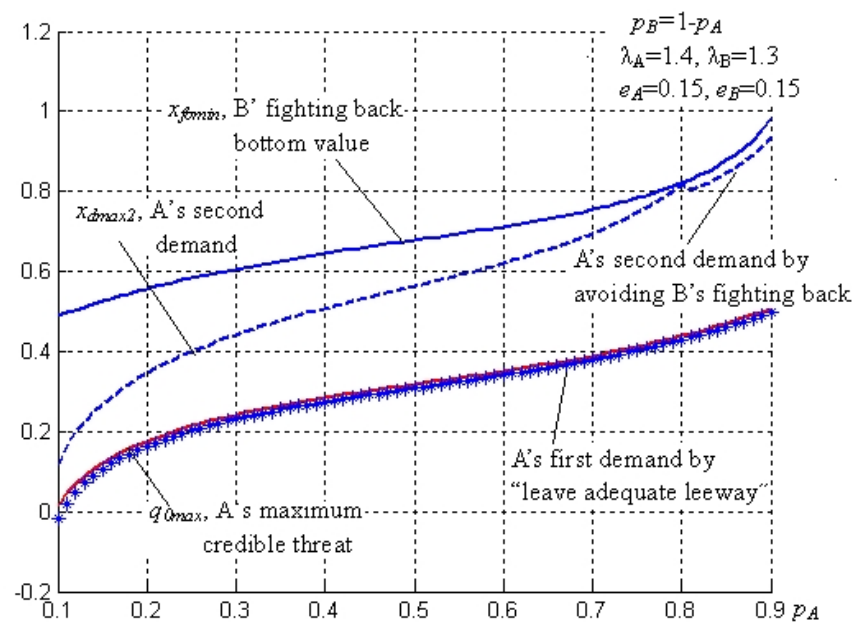

Figure 2. The appearance of "leave adequate leeway " and "fight back"

have nothing at all. By further analysis, it is found that if there are $p_{A}>0.6$ and $p_{B}>0.6$, one more fight will happen because of the loser's attacking. This is the so-called 'Seesaw Battle". When one party's fighting capability is greater than 0.1 and less than 0.6 , the game-playing process is ended by the loser's demanding behavior. Or, the loser loses the ability to fight and has to give up, the process is over.

In Fig.3, "Leave Adequate Leeway" strategy is found once more. When $p_{B}<0.4$, Robot A requests for the first demand $q_{d}<q_{0 \max }$, and then the second for $x_{d \max 2}$. In this way, it can occupy more shares at the game end.

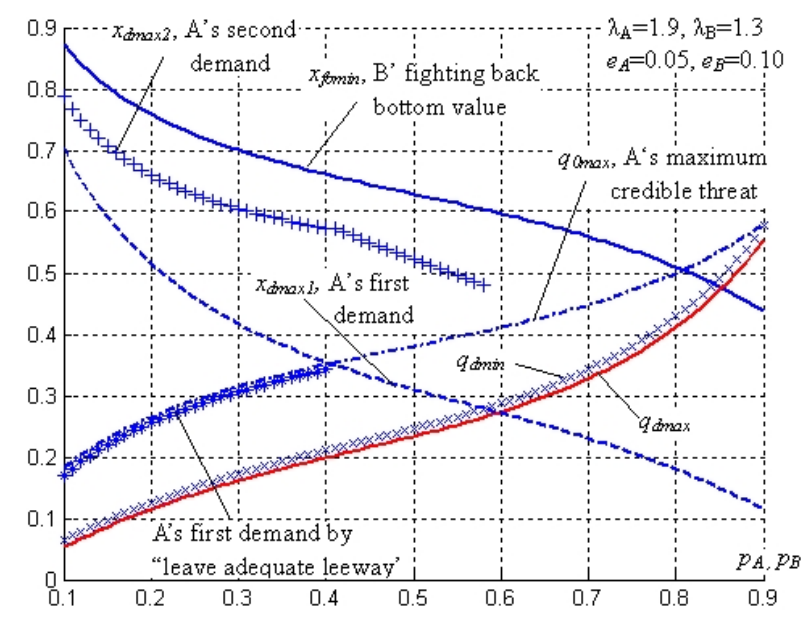

Figure 3. The appearance of "seesaw battle"

\section{Summary}

In this paper, we applied a new emotional decision making mechanism to the emotional game-playing analysis for the coercive bargaining. The careful analyzing process proves the existence and the diversity of emotional game equilibrium. The simulating results are consistent with three Chinese ancient sayings 
on game-playing. Therefore, the suggested emotional game-playing theory is validated. This research shows that the analysis based on driving emotion intensity provides a new approach for the research of emotional affection on game-playing. In order to make game-playing process simply, the feedback emotion is taken into consideration in our study. Therefore, the next step of the research work will focus on the introduction of feedback emotion into game playing.

\section{Acknowledgements}

This work are supported by National Natural Science Foundation of China (No. 61471288、No. 51475351) and PhD early development program of Xi' an technological University (204-000120).

\section{References}

[1] G.F. Zhang, Z.S. Li: Research progress on emotional behavior selection mechanism of artificial life, Journal of Chinese Computer Systems. Vol. 33 (2012) No.8, p. 1774. (In Chinese)

[2] G.F. Zhang: Study on Emotion Driven Behavior Selection Mechanism of Artificial Life (Ph.D., Chongqing University, China, 2009), p. 55. (In Chinese)

[3] L.Z. Wu, G.F. Zhang: Resolution of paradoxes by emotion mechanism, Journal of Anhui University (Philosophy and Social Sciences). Vol. 32 (2008) No.5, p. 46. (In Chinese)

[4] G.F. Zhang, Z.S. Li: Behavior selection mechanism of artificial life based on emotion, Journal of System Simulation. Vol. 21 (2009) No.6, p. 701. (In Chinese)

[5] G.F. Zhang, Z.S. Li: Emotion-based cooperative behavior selection mechanism of artificial life, Information and Control. Vol. 21(2009) No.6, p. 718. (In Chinese)

[6] G.F. Zhang, Z.S. Li: Emotion mechanism of fight behavior, Computer Engineering and Applications. Vol. 45(2009) No.18, p. 28. (In Chinese)

[7] J.Y. Wang, P.P. Li and H.P. Yuan: A critical review on risk decision-making and its attributes, Journal of Engineering Management. Vol. 28(2014) No.1, p. 27. (In Chinese)

[8] G.F. Zhang, N. Wang and H. Xiong: Principe of behavior computing on emotional game, Journal of System Simulation. Vol. 27 (2015) No.1, p. 29. (In Chinese)

[9] C.K. Butler: Prospect theory and coercive bargaining, Journal of Conflict Resolution. Vol. 51 (2007) No.2, p. 227.

[10] C.J. Albers, B.P. Kooi and W. Schaafsma: Trying to resolve the two-envelope problem, Synthese. Vol. 145(2005) No.1, p. 89. 